\title{
Análise de Robustez do Método Híbrido de Estimação de Dano Estrutural
}

L.B.L. SANTOS ${ }^{1}$, H.F. de CAMPOS-VELHO ${ }^{2}$, Instituto Nacional de Pesquisas Espaciais (INPE), 122245-970 São José dos Campos, SP, Brasil.

L.D. CHIWIACOWSKY, Universidade do Vale do Rio dos Sinos (UNISINOS), São Leopoldo, Brasil.

Resumo. Uma análise de robustez de um novo método (método híbrido: algoritmo genético associado a um método de gradiente conjugado) para estimação de dano em estruturas é apresentada. Os testes são realizados na detecção de danos em uma barra, discretizada em elementos finitos.

Palavras-chave. Problemas Inversos em vibração, dano estrutural, método híbrido.

\section{Introdução}

As propriedades mecânicas de uma estrutura podem sofrer mudanças com o passar do tempo, seja por efeitos de uso ou intemperismo. Tais alterações podem comprometer a saúde estrutural do sistema. No problema de identificação de danos assume-se que mudanças nas propriedades de rigidez da estrutura geram alterações detectáveis na resposta vibratória do sistema avaliado. A estratégia de monitoramento e deteç̧ão de dano que tem como base a análise da resposta vibratória (global) do sistema para detectar dano (local) é referida como monitoramento da saúde estrutural (structural health monitoring - SHM) por avaliação não-destrutiva (non-destructive evaluation - NDE) [4].

O problema direto em oscilações mecânicas é caracterizado pelo conhecimento das propriedades mecânicas da estrutura, ex: massa, coeficiente de amortecimento e rigidez, e condições complementares, como condições iniciais e de contorno, tendo como respostas observáveis dinâmicos como deslocamento, velocidade e aceleração.

Um problema inverso é encarado quando tem-se dados como deslocamento ou espectro de freqüências, recolhidos via sensores de precisão e área de cobertura conhecidas, há domínio das condições complementares - experimento controlado e deseja-se saber as propriedades mecânicas, por exemplo, o valor dos componentes da matriz de rigidez global do sistema.

\footnotetext{
${ }^{1}$ Bolsista FAPESP de mestrado - santoslbl@gmail.com

${ }^{2}$ haroldo@lac.br

${ }^{3}$ leondc@gmail.com
} 
O confronto do valor de rigidez, estimado a qualquer tempo, com o apresentado no projeto da estrutura - configuração íntegra - permite inferir a presença de dano estrutural no sistema em avaliação [7] (Figura 1).

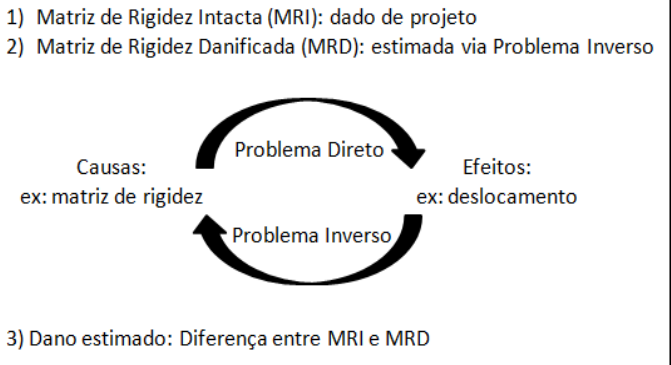

Figura 1: Ilustração da sistemática de estimação de dano estrutural via problema inverso.

Diversos métodos têm sido desenvolvidos para estimação de dano estrutural via problema inverso. O método de Alifanov (formulação variacional resolvida pelo Método de Gradiente Conjugado - MGC), originalmente aplicado à área térmica (problemas de condução do calor) [1], não produz uma solução inversa satisfatória em problemas hiperbólicos - como vibrações mecânicas - nos quais imprecisões nas condições iniciais não são eliminadas ao longo do tempo, o que ocorre em problemas parabólicos, como a transmissão de calor [3].

Em Medeiros (2003) foi proposto um novo operador genético, chamado Epidemia [8]. O operador epidêmico foi usado em Sambatti (2004) [10] no problema inverso de estimação de condição inicial na condução do calor, e foi aplicado com sucesso em outros trabalhos [6].

Em Chiwiacowsky (2005) foi proposta uma estratégia híbrida, onde a estimativa inicial para o método do gradiente conjugado: formulação variacional resolvida via equação adjunta, para a formulação de Alifanov é obtida por um algoritmo genético [4].

A técnica híbrida, que foi pela primeira vez apresentada em Chiwiacowsky et al (2003), foi utilizada em problemas de danos estruturais discretos e contínuos [5] e em um problema de tecnologias espaciais: detecção de danos em Estação Espacial Internacional [6]).

No presente artigo é analisada a robustez de dois métodos de resolução do problema inverso de estimação de dano estrutural frente à quantidade e intensidade do dano: i) MGC, ii) AG + MGC (método híbrido) [11].

A superioridade - maior eficácia sem comprometimento da eficiência - do método híbrido é destacada - bem como sua maior robustez - e propostas de melhorias na operacionalização são proferidas, visando sua aplicação a estruturas de grande porte (alto número de graus de liberdade). 


\section{Metodologia}

A seguir serão detalhados os aspectos de algoritmo e implementação dos métodos aqui utilizados; e é definida a análise de robustez.

Todos testes foram realizados no Cluster Prometeu (pertencente ao Instituto de Física da Universidade Federal da Bahia), que consiste em um sistema HP Blade C7000, com 10 lâminas BL260c, cada uma com a seguinte configuração: 2 processadores Xeon E5405 (2,0 GHz, $1333 \mathrm{FSB})$ com $17 \mathrm{~GB}$ de memória RAM e dois discos SATA de 120 GB. Os tempos de computação de cada método também foram registrados, e comparações absolutas e relativas efetuadas.

\subsection{Aplicação do método dos elementos finitos para deter- minação de matrizes estruturais específicas elementares e globais}

Para discretizar as estruturas cujo dano estrutural deseja-se estimar usou-se o método dos elementos finitos [2]. Como fundamento para a solução de elementos finitos emprega-se o princípio dos trabalhos virtuais. Tal princípio diz que, para assegurar o equilíbrio de um corpo é necessário que para qualquer pequeno deslocamento virtual imposto ao corpo em seu estado de equilíbrio - e compatível com as condições de vínculo - o trabalho virtual interno total deve ser igual ao trabalho virtual externo total: princípio de conservação.

O elemento finito do tipo barra é destinado a sistemas quais apresentam vibrações longitudinais. Cada elemento é caracterizado pela área da seção reta $(A)$, seu comprimento $(l)$ e seu Módulo de Young $(E)$. A matriz de rigidez elementar deste tipo de elemento é dada por

$$
\mathbf{K}^{(e)}=\frac{E A}{l}\left[\begin{array}{cc}
1 & -1 \\
-1 & 1
\end{array}\right] .
$$

Tomemos como exemplo um sistema de 3 elementos finitos, logo 3 graus de liberdade. Na Figura 2 os vetores $u_{1}, u_{2}, u_{3}$ e $u_{4}$ indicam as vibrações longitudinais. Como o sistema está engastado, devemos tomar $u_{1}=0$ (condição de contorno).

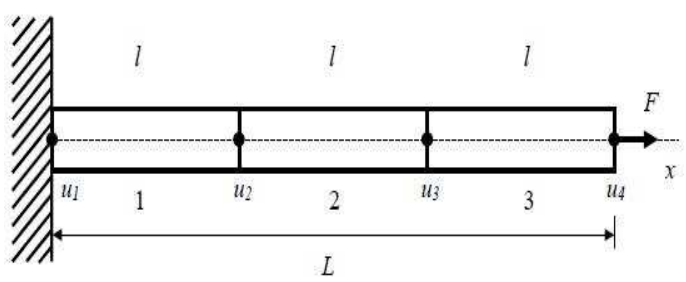

Figura 2: Discretização de uma barra em dois elementos finitos. FONTE: Chiwiacowsky (2005). 
Sobrepondo as matrizes elementares (via blocos na diagonal principal) e aplicando as condições de contorno obtêm-se a matriz de rigidez global - no caso do sistema aqui exemplificado, fica-se com

$$
\mathbf{K}=\frac{E A}{l}\left[\begin{array}{ccc}
2 & -1 & 0 \\
-1 & 2 & -1 \\
0 & -1 & 1
\end{array}\right]
$$

\subsection{Etapas do Algoritmo Genético com o operador epidê- mico}

O Algoritmo Genético (AG) utilizado foi implementado em codificação real, com crossover de ponto simples, cruzamento geométrico e mutação uniforme. Os operadores genéticos utilizados foram os clássicos Seleção, Cruzamento, Mutação e Atualização [8]. O operador epidêmico foi adicionado ao algoritmo original, ficando interno ao laço das iterações - sendo acionado a cada 5 iterações sem alteração na matriz de rigidez estimada.

\subsection{Etapas do Método de Gradiente Conjugado (MGC)}

O Método de Gradiente Conjugado que será aqui utilizado é brevemente descrito a seguir em 9 passos - uma descrição detalhada de cada passo podem ser encontrados em Chiwiacowsky et al, 2005, e maiores detalhes sobre o método geral em [1].

Passo 1: Escolha uma aproximação inicial para K. Tal escolha pode ser feita, por exemplo, tomando-se a matriz de rigidez da estrutura sem dano (dado de projeto).

Passo 2: Resolva o problema direto em qualquer domínio, por exemplo no domínio das frequências, como equação de restrição às possíveis soluções

$$
\left(\mathbf{K}-\lambda_{i} \mathbf{M}\right) \phi_{i}=\mathbf{0}, \quad \text { para } \quad i=1, \ldots, N,
$$

sendo $\mathbf{M}$ a massa do sistema, e obtenha $\lambda$ (espectro de autovalores) e $\mathbf{\Phi}$ (autovetores).

Passo 3: Conhecendo os autovalores calculados $\lambda^{\mathrm{Mod}}$, e os autovalores medidos $\lambda^{\text {Exp }}$, é possível determinar a função custo: distância entre a solução estimada e a proveniente do dado experimental. Tais soluções são funções do parâmetro a ser estimado, no caso, da matriz de rigidez. A distância utilizada no caso aqui apresentado foi a Euclidiana. O problema é levado ao domínio dos multiplicadores de Lagrange $(\psi)$, e é então resolvido o problema adjunto

$$
\psi=2 \Phi\left[\lambda^{\mathrm{Mod}}-\lambda^{\mathrm{Exp}}\right] .
$$

Passo 4: Conhecendo $\psi$, calcule a função gradiente através de

$$
\nabla J_{j}(\mathbf{K})=\phi^{T} \triangle \mathbf{K}_{j} \psi, \quad \text { para } j=1, \ldots, N_{e} .
$$


Passo 5: Calcule o coeficiente conjugado $\gamma$

$$
\gamma=\frac{\left\|\nabla J^{k+1}\right\|_{2}^{2}}{\left\|\nabla J^{k}\right\|_{2}^{2}} .
$$

Passo 6: Calcule a direção descendente $\boldsymbol{p}$

$$
\boldsymbol{p}^{0}=-\nabla J\left(\mathbf{K}^{0}\right), \boldsymbol{p}^{k+1}=-\nabla J\left(\mathbf{K}^{k+1}\right)+\gamma \boldsymbol{p}^{k} .
$$

Passo 7: Resolva o problema de sensibilidade e obtenha o comprimento descendente $\boldsymbol{\beta}$, que minimiza a seguinte função escalar

$$
\phi(r)=J\left(\mathbf{K}^{k}+r \boldsymbol{p}^{k}\right), \quad \text { para } \quad k=0,1, \ldots
$$

Passo 8: Calcule a correção para $\mathbf{K}$

$$
\mathbf{K}^{k+1}=\mathbf{K}^{k}+\beta^{k} \boldsymbol{p}^{k}, \quad \text { para } \quad k=0,1, \ldots .
$$

Passo 9: Encerre o ciclo iterativo quando o critério de parada [9],

$$
J(\mathbf{K})<\epsilon^{2},
$$

for satisfeito. Caso contrário, volte ao Passo 2. $\epsilon$ pode ser encarado como o nível de ruído presente nos dados. Em todas as simulações aqui efetuadas usou-se $\epsilon=5 E-2: 5 \%$ de ruído gaussiano multiplicativo no dado sintético.

\subsection{Análise de robustez}

O resultado do programa que será a base para a comparação entre métodos é uma análise de robustez: análise do erro de estimação do dano em função do número de elementos danificados e intensidade do dano. Vale ressaltar que essa análise não foi antes efetuada em nenhum dos métodos, em nenhum sistema avaliado.

O erro de estimação é definido como a média, para todos elementos finitos, do módulo da diferença entre a porcentagem de dano real e estimado. O resultado é apresentado na forma de uma matriz, na qual as linhas representam a intensidade do dano (porcentagem, iniciando em $5 \%$ e indo até $95 \%$ com passo de $5 \%$ ), as colunas o número de elementos danificados (de 1 a 9 , sendo no total 10 elementos finitos) e os elementos da matriz correspondendo ao erro de estimação. Uma vez que o AG é um método probabilístico, as simulações são efetuadas para um número pré-fixado de amostras (ex: 20) e são apresentados os resultados de erro médio e mínimo. Além disso, dependendo da escolha da condição inicial, o MGC aqui implementado pode abortar: executar mais de uma vez a metodologia permite uma maior probabilidade de obter um resultado - neste caso analisa-se o resultado também para um número pré-fixado de amostras, ex: 20 - a execução não única do método provê diferentes cenários para a avaliação do dano, todos com possibilidade de avaliação experimental (inspeção). 


\section{Resultados e Discussões}

Uma sequência de resultados pertinentes à análise de robustez é apresentada a seguir: Figura 3. A cor de cada retângulo nas figuras corresponde ao valor do erro de estimação do dano - valor $(\mathrm{i}, \mathrm{j}$ ) - no sistema de i elementos danificados, com dano de intensidade j. De preto a branco são apresentados 20 tons de cinza, cada tom correspondendo a um intervalo de $5 \%$ de erro de estimação. Em branco os resultados que apresentaram erro de estimação superior a 100\% (ex: o dano real é $20 \%$ e o método estimou mais de $120 \%$ - erro grosseiro).

O resultado referente às médias (Figuras $3 \mathrm{a}$ e 3c) permite dizer que a hibridização confere à metodologia de estimação de dano estrutural melhores resultados, em média, em casos de poucos elementos danificados, mesmo com alta intensidade do dano. Todavia, para muitos elementos com dano (de baixa intensidade) o MGC acaba apresentando, em média, melhores resultados.

Em aplicações práticas, entretanto, não há como saber o erro médio de estimação: essa é uma grandeza de teste do método, logo o que será usado pela equipe de teste (inspeção direta) de dano é o resultado referente a erro mínimo: uma vez que os diferentes cenários de possíveis danos serão todos avaliados, e então o cenário de erro mínimo será realmente determinado. O confronto entre os diferentes métodos aqui apresentados deve, portanto, ser efetuado com base nos resultados de erro mínimo (Figuras 3b e 3d).

No MGC percebe-se que para intensidades de dano até $20 \%$, não importando o número de elementos danificados, os resultados são bons. Para intensidades de dano superiores a 40\%, mesmo com poucos elementos danificados, o método não apresenta resultados razoáveis: os erros de estimação são altos. Na região entre 20 e $40 \%$ de intensidade de dano os resultados são bons apenas para um pequeno número de elementos danificados.

Para o método híbrido nota-se que até uma intensidade de dano da ordem de $50 \%$, na grande maioria dos casos, acarreta bons resultados. Para danos mais intensos que $70 \%$ do valor original de rigidez os resultados não são razoáveis mesmo para poucos elementos danificados.

Para cada figura há, portanto, a possibilidade de traçar-se 3 regiões tendo como separatrizes valores de intensidade limite de dano: na $1^{\mathrm{a}}$ os resultados são bons, em média, independentemente do número de elementos danificados; na $2^{\text {a }}$ a qualidade dos resultados é função decrescente do número de elementos com dano; e na $3^{\mathrm{a}}$ região os resultados não são razoáveis, mesmo para poucos elementos danificados. Para o MGC as separatrizes são 20 e $40 \%$ e para o AG + MGC são 50 e $70 \%$.

A hibridização não apenas melhorou a média global de estimação do erro varrendo todas as linhas e colunas das matrizes de robustez - (32\% para o MGC e $17 \%$ para o métod híbrido), mas também reduziu o limitante superior do erro - tudo isso sem comprometer efetivamente a eficiência, o tempo de obtenção do resultado - tempo de computação da solução (53 milisegundos para o MGC e 55 milisegundos para o método híbrido). O método híbrido já começa com uma solução (solução iterativa inicial), em média, melhor que uma condição aleatória (como no caso do MGC), este início mais próximo à bacia do mínimo global provê uma maior probabilidade para o método híbrido de apresentar uma solução final mais próxima 
à solução desejada.

a)
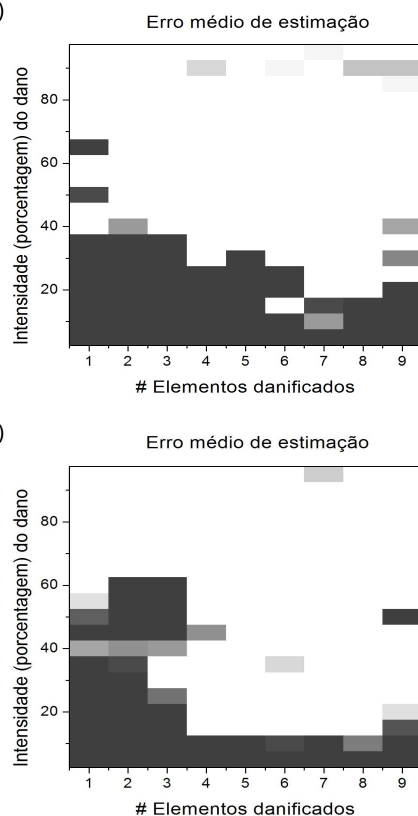

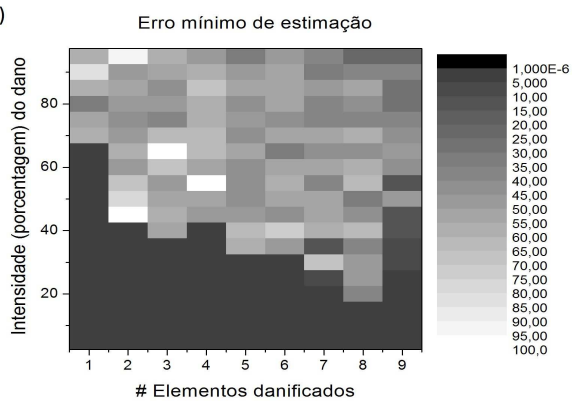

d)

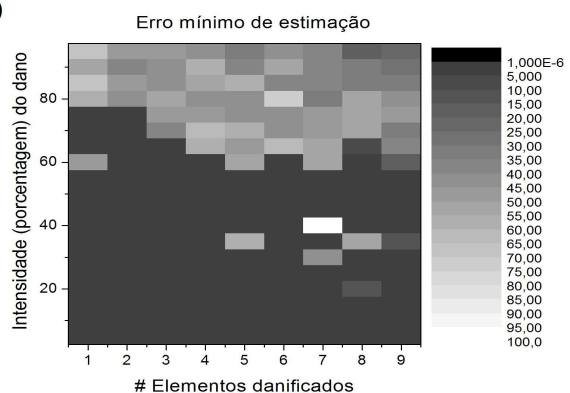

Figura 3: a) Erro de estimação médio para o MGC, b) Erro de estimação médio para o método híbrido, c) Erro de estimação mínimo para o MGC, d) Erro de estimação mínimo para o método híbrido. A cor de cada retângulo corresponde ao valor do erro de estimação do dano - valor $(\mathrm{i}, \mathrm{j})$ - no sistema de i elementos danificados, com dano de intensidade j. De preto a branco são apresentados 20 tons de cinza, cada tom correspondendo a um intervalo de $5 \%$ de erro de estimação.

\section{Conclusões e Perspectivas}

Resultados originais frente à robustez do método híbrido de estimação de dano estrutural foram aqui apresentados. O Método de Gradiente Conjugado (MGC) como originalmente formulado não produz normalmente soluções inversas satisfatórias em problemas hiperbólicos, devido à não eliminação de incertezas (flutuações, ruído) nas condições iniciais. A hibridização resolve tal problema, e o método híbrido por já começar com uma solução, em média, melhor que uma condição aleatória - como no caso do MGC convencional - apresenta uma solução final mais próxima à solução desejada.

O erro médio global de estimação mostra que a hibridização não apenas melhora a média global de estimação do erro, mas também reduziu o limitante superior do erro - tudo isso sem comprometer efetivamente a eficiência, o tempo de obtenção do resultado - tempo de computação da solução. 
Dentre as perspectivas desta investigação está a aplicação da análise de robustez a outras estruturas: tipo treliça e tipo viga, e então avaliar se o método é geral o bastante para apresentar bons resultados indepentemente do tipo de sistema físico em análise.

Abstract. A robustness analysis of a new method (hybrid method: genetic algorithm associated with a conjugated gradient method) to estimate structural damage is presented. The tests are performed to detect damage in a beam, discretized in finite elements.

\section{Referências}

[1] O.M. Alifanov, Solution of an inverse problem of heat conduction by iteration methods, Journal of Engineering Physics, 26 (1974), 471-476.

[2] K. Bathe, "Finite Element Procedures", Prentice-Hall, New Jersey, 1996.

[3] H.F. de Campos Velho, "Problemas Inversos em Pesquisa Espacial", Mini-curso, Congresso Nacional de Matemática Aplicada e Computacional (CNMAC), Belém (PA), Brasil, 2008.

[4] L.D. Chiwiacowsky, "Método Variacional e Algoritmo Genético em Identificação de Danos Estruturais", Tese de Doutorado em Computação Aplicada, INPE, São José dos Campos, Brasil, 2005.

[5] L.D. Chiwiacowsky, H.F. de Campos Velho, P. Gasbarri, The damage identification problem: a hybrid approach. Vol. 2, Anais do DINCON 2003, 2003.

[6] L.D. Chiwiacowsky, P. Gasbarri, H.F. de Campos Velho, Damage assessment of large space structures through the variational approach. Acta Astronautica, 62, No. 10-11, (2008), 592-604.

[7] S. Doebling, C. Farrar, M. Prime, D. Shevitz, Damage identification and health monitoring of structural and mechanical systems from changes in their vibration characteristics: a literature review. Technical Report. Los Alamos, USA, 1996.

[8] F.L.L. Medeiros, "Algoritmo Genético Híbrido como um Método de Busca de Estados Estacionários de Sistemas Dinâmicos", Dissertação de Mestrado em Computação Aplicada, INPE, São José dos Campos, Brasil, 2003.

[9] V. Morozov, M. Stessin, "Regularization methods for ill-posed problems", CRC Press, New York, 1993.

[10] S. Sambatti, "Diferentes Estratégias de Paralelização de um Algoritmo Genético Epidêmico Aplicadas na Solução de Problemas Inversos em Transferência de Calor", Dissertação de Mestrado em Computação Aplicada, INPE, São José dos Campos, Brasil, 2004.

[11] L.B.L. Santos, "Abordagem Hierárquica ao Método Híbrido de Estimação de Danos em Estruturas Aeroespacias", Dissertação de mestrado em Computação Aplicada, INPE, São José dos Campos, Brasil, 2011. 